\title{
Evidencia sobre las vacunas para COVID-19 en niñas, niños y adolescentes
}

\author{
Evidence on vaccines for COVID-19 in children and adolescents
}

Agostina Risso $^{\mathrm{a}}(\mathbb{D})$, Malena Chiaborelli ${ }^{\mathrm{a}}(\mathbb{D})$ Agustín Ciapponia $^{\mathrm{a}, \mathrm{b}}(\mathbb{D})$

\begin{abstract}
Resumen
En Argentina, tres de las vacunas contra el COVID-19 autorizadas por la entidad regulatoria local se aplican en menores de edad: la vacuna desarrollada por Sinopharm (BBIBP-CorV) para niños de 3 años o más, y las desarrolladas por Moderna (ARNm 1273) y Pfizer (BNT162b2) a partir de los 12 años. Dado que estas recomendaciones no coinciden exactamente con las emitidas por diferentes organismos internacionales y debido al corto plazo de seguimiento de los ensayos publicados, surgieron dudas fundamentalmente en relación a su seguridad. En este artículo, los autores sintetizan la evidencia disponible hasta el momento sobre las vacunas aplicadas en niños, niñas y adolescentes en nuestro país, basada en informes preliminares de ensayos clínicos y reportes de vigilancia epidemiológica.

Abstract

In Argentina, three of the COVID-19 vaccines authorized by the local regulatory entity are applied to minors: the vaccine developed by Sinopharm (BBIBP-CorV) for children 3 years of age or older, and those developed by Moderna (RNAm 1273) and Pfizer (BNT162b2) from the age of 12 and onwards. Given that these recommendations do not coincide exactly with those issued by different international organizations and due to the short follow-up period of the published trials, doubts arose fundamentally in relation to their safety. In this article, the authors summarize the evidence available to date on vaccines applied to children and adolescents in our country, based on preliminary studies of clinical trials and epidemiological surveillance reports.
\end{abstract}

Palabras clave: Infecciones por Coronavirus, COVID-19, Vacunas contra la COVID-19. Keywords: Coronavirus Infections, COVID-19, COVID-19 Vaccines

Risso A, Chiaborelli M, Ciapponi A. Evidencia sobre las vacunas para COVID-19 en niñas, niños y adolescentes. Evid Actual Pract Ambul. 2022;25(1):e006997. Available from: https://dx.doi.org/10.51987/EVIDENCIA.V25I1.6997.

\section{Introducción}

Desde el inicio de la pandemia de COVID-19 se ha observado que los casos en niños, niñas y adolescentes han sido menos frecuentes y menos graves que en los adultos. Aun así, esta población no ha estado exenta de padecer formas severas de la enfermedad ${ }^{1}$, jugando además un importante rol en la transmisión viral ${ }^{2}$. Por otra parte, no caben dudas de que el aislamiento social preventivo y obligatorio prolongado dispuesto para contener la pandemia afectó su desarrollo social y educativo, además de agobiar a sus cuidadores. De hecho, ha habido un gran debate social y científico en torno a la presencialidad escolar ${ }^{3}$. Es así que la vacunación contra el SARS-CoV-2 en este grupo etario se postula como una estrategia clave, desde varios aspectos, para hacer frente a la pandemia.

Organismos internacionales han emitido distintas recomendaciones respecto a las edades de indicación de cada vacuna. Por ejemplo, la Organización Mundial de la Salud (OMS) solo recomienda la vacuna Pfizer-BioNTech (BNT162b2) a partir de los 12 años de edad, mientras que el resto de las vacunas que cuentan con su aval están indicadas en adultos mayores de 18 años ${ }^{4}$. La Administración de Medicamentos y Alimentos del Gobierno de los Estados Unidos (FDA, por sus iniciales en inglés) y los Centros para el Control y la Prevención de Enfermedades (CDC, por sus iniciales en inglés), también recomiendan solo esta vacuna para los niños, pero a partir de los 5 años ${ }^{5-7}$. En cambio, la Agencia Europea de Medicamentos (EMA, por sus iniciales en inglés) recomienda tanto la vacuna Pfizer-BioNTech (BNT162b2) como la desarrollada por Moderna (RNAm 1273) para niños de 12 años en adelante ${ }^{8,9}$
Al día de la fecha, las vacunas autorizadas por la Agencia Nacional de Medicamentos, Alimentos y Tecnología Médica (ANMAT) en Argentina para menores de edad incluyen la desarrollada por Sinopharm (BBIBP-CorV) a partir de los 3 años y las desarrolladas por Moderna (ARNm 1273) y Pfizer-BioNTech (BNT162b2), a partir de los 12 años ${ }^{10,11}$. En los últimos días, este organismo emitió además la autorización para que una nueva formulación de la vacuna BNT162b2 sea aplicada en niños y niñas de 5 a 11 años ${ }^{12}$, en base a los resultados de un informe interino recientemente publicado ${ }^{13}$, y se espera que pronto esté disponible en este país ${ }^{14}$.

Ante las discordancias observadas en las recomendaciones surgieron debates en torno a la vacunación -quizás más políticos que científicos-, por lo que nos propusimos analizar la evidencia que sustenta las vacunas que se encuentran aprobadas y en uso en la actualidad para los niños, niñas y adolescentes de Argentina.

\section{Resumen de la evidencia}

En este artículo sintetizamos tres informes interinos de ensayos clínicos aleatorizados, doble ciegos, comparados contra placebo, que continúan en marcha, en los que se evalúa la seguridad, inmunogenicidad y eficacia de las vacunas contra el SARS CoV-2 en niños, niñas y adolescentes sanos.

Para el caso de la vacuna Pfizer-BioNTech (BNT162b2), analizamos un informe que evaluó los efectos de la vacuna en población de adolescentes sanos de 12 a 15 años de edad en los EE.UU. ${ }^{15}$, comparando los datos obtenidos con la misma vacuna en la población de adultos jóvenes de 16 a 25 años.

\footnotetext{
a Servicio de Medicina Familiar y Comunitaria, Hospital Italiano de Buenos Aires. agostina.risso@hospitalitaliano.org.ar, malena.chiaborelli@hospitalitaliano.org.ar,

b Centro Cochrane Argentina, Instituto de Efectividad Clínica y Sanitaria. aciapponi@iecs.org.ar
} 
En el caso de la vacuna de Moderna (ARNm 1273), resumimos un informe interino con los resultados de la primera fase del ensayo Teen COVE ${ }^{16}$, que evalúa la vacuna en la población de adolescentes sanos de 12 a 17 años del mismo país, y compara sus resultados con los obtenidos en el ensayo COVE en población adulta.

Tabla 1. Eficacia, inmunogenicidad y seguridad de las vacunas COVID-19 en niños y adolescentes disponibles en Argentina. Notas: IC: intervalo de confianza; GMR: razón media geométrica. *En los grupos de 3 a 5, 6 a 12 , y 13 a 17 años, respectivamente. **Ninguno de estos dos efectos adversos fue significativamente diferente entre los vacunados y los controles.

\begin{tabular}{|c|c|c|c|}
\hline Vacuna & $\begin{array}{l}\text { Pfizer-BioNTech } \quad(B N T 162 b 2) \\
(12 \text { a } 17 \text { años })^{15}\end{array}$ & $\begin{array}{l}\text { Moderna (ARNm 1273) (12 a } \\
17 \text { años) }{ }^{16}\end{array}$ & $\begin{array}{l}\text { Sinopharm (BBIBP-CorV) }(3 \\
\text { a } 17 \text { años })^{17}\end{array}$ \\
\hline Tamaño muestral & 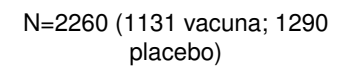 & $\begin{array}{l}\mathrm{N}=3732 \text { (2489 vacuna; } 1243 \\
\text { placebo) }\end{array}$ & $\begin{array}{l}\mathrm{N}=1008 \text { (756 vacuna; } 252 \\
\text { placebo) }\end{array}$ \\
\hline $\begin{array}{l}\text { Prevención COVID-19 (infección sintomática) } \\
\text { luego de } 2 \text { dosis }\end{array}$ & $100 \%($ IC $95 \% 75,3$ a 100$)$ & $93,3 \%$ (IC $95 \% 47,9$ a $99,9 \%)$ & No disponible \\
\hline GMR de anticuerpos neutralizantes & $1,76$ (IC $95 \% 1,47$ a 2,1$)$ & $1,08($ IC $95 \% 0,94$ a 1,24$)$ & $1,04$ (IC $95 \% 0,93$ a 1,18$)$ \\
\hline \multirow[t]{2}{*}{ Eventos adversos locales } & \multicolumn{3}{|c|}{ Dolor en el sitio de inyección } \\
\hline & 79 a $86 \%$ & $93 \%$ & $4 \%, 9,1 \%$ y $7,9 \% *$ \\
\hline Eventos adversos sistémicos & \multicolumn{2}{|c|}{ Fatiga y cefalea } & Fiebre y tos ** \\
\hline Eventos adversos serios & \multicolumn{3}{|c|}{$\begin{array}{c}\text { No hubo eventos adversos serios relacionados con la vacuna. No se registraron casos de } \\
\text { miocarditis, pericarditis ni muertes }\end{array}$} \\
\hline
\end{tabular}

\section{Eficacia}

Para evaluar la eficacia de la vacuna para prevenir la enfermedad (sintomática) por COVID fueron comparados el porcentaje de participantes vacunados que contrajeron la enfermedad con el porcentaje de participantes no vacunados que contrajeron COVID-19.

En el estudio de la vacuna Moderna (ARNm 1273), fue evaluada además su eficacia para prevenir la infección asintomática por SARS-CoV-2 en adolescentes, la cual fue de 39,2\% (Intervalo de confianza [IC] del $95 \% 24,7$ a 69,7$)^{16}$.

\section{Inmunogenicidad}

Los estudios de Pfizer-BioNTech (BNT162b2) y Moderna (ARNm 1273) realizaron un análisis de no inferioridad en el que compararon la razón de la media geométrica del título de anticuerpos neutralizantes alcanzados al mes de la segunda dosis en niños y adolescentes vacunados, en comparación con los alcanzados en los adultos vacunados. Como punto de corte para cumplir con el criterio de no inferioridad se estableció un límite inferior del IC del $95 \%$ mayor a 0,67. Como se observa en la Tabla 1 , en ambos casos este umbral fue alcanzado, con una mejor respuesta inmunogénica en la población adolescente que en la población adulta en el caso de la vacuna BNT162b2.

En el estudio de la vacuna desarrollada por Sinopharm (BBIBPCorV), fueron comparados los títulos de anticuerpos alcanzados en las personas vacunadas y no vacunadas y, además, estos títulos con el nivel de anticuerpos presente en el plasma de convalecientes. Los autores observaron un aumento sustancial en los títulos de anticuerpos luego de la segunda dosis de la vacuna (más marcado con las dosis de $4 \mathrm{mcg}$, usada en Argentina, y de $8 \mathrm{mcg}$ ), con valores similares a los alcanzados en el suero de convalecientes. Además, fue documentado que el $100 \%$ de los participantes alcanzó la seroconversión a los 28 días de la segunda dosis de esta vacuna.
Del mismo modo, presentamos los resultados preliminares de la vacuna Sinopharm (BBIBP-CorV) comparando distintos esquemas y dosis de la vacuna en distintos grupos etarios de niños y adolescentes sanos de China de 3 a 17 años ${ }^{17}$.

\section{Resultados}

La Tabla 1 presenta los principales efectos de las vacunas evaluadas en este artículo. miocarditis, pericarditis ni muertes

\section{Seguridad}

La mayoría de los eventos adversos reportados fueron leves a moderados, remitieron espontáneamente luego de uno o dos días y fueron similares entre las distintas vacunas y grupos etarios. A nivel local, el dolor en el sitio de inyección fue el efecto adverso más frecuente, y a nivel sistémico, la fatiga y la cefalea. En ninguno de los informes fueron documentados eventos adversos serios relacionados con la vacuna, casos de miocarditis, pericarditis ni muertes.

\section{Limitaciones}

Entre las principales limitaciones de los tres estudios analizados podemos destacar el corto plazo de seguimiento, que alcanzó un máximo de dos meses luego de la segunda dosis. Serán necesarios estudios con periodos mayores de seguimiento tanto para determinar la persistencia de anticuerpos y la eficacia como para evidenciar eventos adversos menos frecuentes.

Por otra parte, las poblaciones de los estudios fueron poco diversas, con un bajo porcentaje de población latina, y solo el estudio de Moderna (ARNm 1273) evaluó la eficacia de la vacuna para prevenir la infección viral asintomática.

\section{Algunas consideraciones sobre la seguridad de las vacunas en Argentina}

De acuerdo al informe elaborados por la Dirección de Control de Enfermedades Inmunoprevenibles (DiCEI) del Ministerio de Salud de la Nación en conjunto con la Comisión Nacional de Seguridad en Vacunas (CoNaSeVa) acerca de la seguridad de la vacunación contra el COVID-19 en niños, niñas y adolescentes en Argentina, los eventos supuestamente atribuibles a la vacunación o inmunización (ESAVI) pueden definirse como cualquier situación de salud no esperada (signo no favorable o no intencionado, hallazgo anormal de laboratorio, síntoma o enfermedad) que ocurre posterior a la vacunación y que no necesariamente tiene una relación causal con ésta. Es decir, que son eventos que se 
encuentran temporalmente relacionados con la vacunación, pero no necesariamente son causados por la misma, por lo que se denominan 'supuestamente atribuibles a la vacunación' 18.

Este informe incluye los datos reportados desde el inicio de la campaña de vacunación contra el SARS-CoV-2 en adolescentes (28 de julio del 2021) y en niños y niñas de entre 3 y 11 años (12 de octubre del 2021 hasta el 31 de octubre del 2021) ${ }^{18}$, y aña- de a los datos provenientes de los ensayos clínicos (limitados por su breve periodo de seguimiento), valiosa información de seguridad en relación a los posibles efectos adversos de las vacunas analizadas en este artículo.

La Tabla 2 presenta las tasas de notificación de ESAVI y ESAVI graves cada 100.000 dosis aplicadas en Argentina, desagregadas por vacuna.

Tabla 2. Dosis aplicadas, ESAVI notificados y tasa de notificación de ESAVI cada 100.000 dosis aplicadas, según vacuna contra el COVID-19 en adolescentes (12 a 17 años) y niños y niñas (3 a 11 años). Nota: ESAVI: eventos supuestamente atribuibles a la vacunación o inmunización. Fuente: Ministerio de Salud de la Nación y CoNaSeVa, en base a la información reportada en el módulo ESAVI (SIISA) y el Registro Nominal de Vacunación (NOMIVAC) al 31/10/2021.

\begin{tabular}{|l|c|c|c|c|}
\hline Vacuna & Dosis aplicadas & ESAVI $(\mathrm{n})$ & Tasa de ESAVI / 100.000 dosis & Tasa de ESAVI graves / 100.000 dosis \\
\hline Sinopharm (BBIBP-CorV) & 1.932 .165 & 153 & 7,9 & 1,2 \\
\hline Moderna (ARNm 1273) & 1.055 .244 & 326 & 30,9 & 2,7 \\
\hline Pfizer-BioNTech (BNT162b2) & 1.741 .476 & 158 & 9,1 & 1,1 \\
\hline Total & 4.728 .885 & 637 & 13,5 & 1,5 \\
\hline
\end{tabular}

Los eventos más frecuentes clasificados como relacionados a la vacunación según la clasificación de la OMS fueron reacciones locales con o sin fiebre, síndrome pseudogripal, cefalea, mialgias, artralgias, astenia, escalofríos y alergia.

De los eventos graves atribuibles a la vacuna de Sinopharm (BBIBP-CorV), tres se consideraron coincidentes (no relacionado a la vacunación) y uno como ansiedad a la vacunación (episodio sincopal con posterior traumatismo que requirió observación en guardia). En referencia a la vacuna Pfizer-BioNTech (BNT162b2), dos eventos graves fueron clasificados como ansiedad (episodios sincopales) y un evento como coincidente, mientras que en el caso de Moderna (ARNm 1273), nueve fueron clasificados como coincidentes (no relacionados a la vacunación), dos como no clasificables por falta de información y uno como relacionado al producto (alergia grave que requirió observación en guardia, con recuperación ad integrum). El resto de los eventos graves para las tres vacunas (entre los que se encuentra una miocarditis con restitución ad integrum posterior a la aplicación de BNT162b2) permanece bajo análisis.

En relación a la preocupación por los casos de miocarditis o pericarditis luego de la aplicación de las vacunas a ARN mensajero (BNT162b2 y ARNm 1273), el Comité Asesor de Prácticas de Inmunización del CDC ha comunicado una tasa estimada de incidencia en hombres de 12 a 29 años es de 41 casos por millón luego de la segunda dosis (en su gran mayoría, casos leves), por lo que concluye que los beneficios de la vacunación superan ampliamente los riesgos ${ }^{19}$. Esta postura fue avalada por la Sociedad Argentina de Cardiología en una misiva publicada días atrás por la Sociedad Argentina de Pediatría (SAP) 20

En su comunicado, la SAP respalda además la vacunación contra SARS-Cov-2 en niñas, niños y adolescentes al sostener cuatro puntos nodales que resumimos:

1. Carga de enfermedad: el riesgo existe. La población pediátrica representa el $8,8 \%$ del total de los casos confirmados desde el inicio de la pandemia en nuestro país, y en la actualidad, uno de cada cuatro casos confirmados ocurre en menores de 18 años. Aunque con poca frecuencia se presentan cuadros respiratorios moderados o graves, existe una nueva (y seria) entidad denominada SIMC (Síndrome Inflamatorio Multisistémico temporalmente relacionado a COVID-19), que aparece de 2 a 6 semanas después de haber padecido la infección por SARS-CoV-2. Otro aspecto a considerar son las secuelas a largo plazo que pueden desarrollar hasta el $10 \circ 15 \%$ de los niñas, niños y adolescentes que han padecido COVID-19.

2. Características de la transmisibilidad y oportunidad de integración a actividades. Este grupo etario puede transmitir el virus a otras personas, aún considerando las adecuadas coberturas de vacunación contra COVID-19 en adultos. La protección activa con vacunas seguras y eficaces permitirá, además, proteger a los niños frente a la emergencia de nuevas variantes, que parecen propagarse con mayor facilidad en la población pediátrica. La vacunación pediátrica permitiría entonces: proteger a las familias, facilitar una presencialidad escolar más segura, permitir otras actividades deportivas, lúdicas y sociales de los niños y disminuir la ansiedad y preocupación de los padres.

3. Inmunidad colectiva. Todas las vacunas contra la COVID-19 en uso en nuestro país contienen virus inactivados o material genético viral, por lo que no tienen la capacidad de inducir inmunidad a nivel de las mucosas, como ocurre con otras con plataformas de virus atenuados. Es por ello fundamental alcanzar a nivel poblacional una alta cobertura de vacunación que construya una barrera epidemiológica para dificultar la transmisión de SARS-CoV-2 y lograr inmunidad colectiva. Resalta el concepto de solidaridad social, en el que gracias a la vacunación colectiva existe una menor circulación viral y, por lo tanto, menor posibilidad de enfermarse, beneficiándose no sólo aquellos exclusivamente vacunados.

4. Vacunas disponibles. Todas las vacunas contra SARS Cov-2 usadas en nuestro país fueron aprobadas o autorizadas por la ANMAT. Las vacunas aplicadas en nuestro país contra el COVID-19 son seguras y efectivas.

El comunicado de la SAP remarca que, si bien evaluar la efectividad de una vacuna es extremadamente complejo, en los países con alta tasa de vacunación se ha visto una clara disminución en la cantidad de contagios y, sobre todo, una disminución de la mortalidad, por lo que concluye que, si bien es muy necesario mantener una cuidadosa vigilancia de la seguridad y eficacia de 
las vacunas en uso, también lo es seguir avanzando con la vacunación en niños, niñas y adolescentes.

\section{Conclusiones de los autores}

La evidencia presentada, proveniente de ensayos clínicos aleatorizados y de datos de vigilancia a nivel nacional, sostiene que las tres vacunas disponibles en Argentina son seguras e inmunogénicas durante el período de seguimiento disponible a la fecha. Además, las publicaciones sobre las vacunas desarrolladas por Pfizer-BioNTech (BNT162b2) y Moderna (ARNm 1273) mostraron eficacia para prevenir la enfermedad por COVID-19, y la última incluso documentó cierta eficacia en la prevención de infección asintomática.
Si bien la calidad de la evidencia no es la que se exigiría en condiciones ideales, dada la situación de emergencia sanitaria, la toma de decisiones sanitarias debe realizarse en base a la mejor evidencia disponible y, a la luz de las problemáticas emergentes, estas deben ir ajustándose de forma permanente, en base a la evidencia acumulativa y a una rigurosa vigilancia epidemiológica.

Las diferencias de criterio entre los distintos organismos internacionales parecen ser menores en relación a la seguridad y al contundente beneficio demostrado por las vacunas en los distintos ensayos clínicos y en los estudios observacionales.

Recibido el 23/12/2021 Aceptado el 21/01/2022 y Publicado el 25/01/2022.

\section{Referencias}

1. Tsabouri S, Makis A, Kosmeri C, et al. Risk factors for severity in children with coronavirus disease 2019: a comprehensive literature review. Pediatr Clin North Am. 2021;68(1):321-338. Available from: 10.1016/j.pcl.2020.07.014.

2. Moghadas SM, Fitzpatrick MC, Shoukat A, et al. Simulated Identification of Silent COVID-19 Infections Among Children and Estimated Future Infection Rates With Vaccination. JAMA Netw Open. 2021;4(4):e217097. Available from: 10.1001/jamanetworkopen.2021.7097.

3. Ciapponi A. La suspensión de la presencialidad escolar: un resumen de la evidencia disponible a nivel internacional respecto de su efectividad para contener la actual pandemia de COVID-19. Evid Actual Pract Ambul. 2021;24(2):e002131. Available from: 10.51987/evidencia.v24i3.6950.

4. Organización Mundial De La Salud. Enfermedad por el Coronavirus (COVID-19): Vacunas;. Available from: https://www.who.int/emergencies/ diseases/novel-coronavirus-2019/covid-19-vaccines [Last access: 2022-01-24].

5. U S Food and Drug Administration. La FDA autoriza vacuna contra el COVID-19 de Pfizer-BioNTech para uso de emergencia en niños de 5 a 11 años de edad; 2021. Available from: https:/www.fda.gov/news-events/press-announcements/la-fda-autoriza-vacuna-contra-el-covid-19-de-pfizerbiontech-para-uso-de-emergencia-en-ninos-de-5-11 [Last access: 2022-01-24].

6. U S Food and Drug Administration. Información sobre las vacunas para el COVID-19. Vacunas contra el COVID-19 autorizadas para uso de emergencia o aprobadas por la FDA; 2021. Available from: https://www.fda.gov/about-fda/fda-en-espanol/informacion-sobre-las-vacunas-para-el-covid-19 [Last access: 2022-01-24].

7. Centros para el Control y la Prevención de Enfermedades. Vacunas contra el COVID-19 para niños y adolescentes; 2021. Available from: https: //espanol.cdc.gov/coronavirus/2019-ncov/vaccines/recommendations/children-teens.html [Last access: 2022-01-24].

8. Agencia Europea De Medicamentos. COVID-19 vaccine Spikevax approved for children aged 12 to 17 in EU; 2021. Available from: https://www.ema. europa.eu/en/news/covid-19-vaccine-spikevax-approved-children-aged-12-17-eu [Last access: 2022-01-24].

9. European Vaccination Information Portal. Covid-19 vaccines; 2021. Available from: https://vaccination-info.eu/en/covid-19/covid-19-vaccines [Last access: 2022-01-24].

10. Argentina Ministerio de Salud de la Nacion. Preguntas frecuentes sobre la vacuna contra COVID-19; 2021. Available from: https://www.argentina. gob.ar/coronavirus/vacuna/preguntas-frecuentes [Last access: 2022-01-24].

11. Argentina Ministerio de Salud de la Nacion. Preguntas frecuentes pediátricas;. Available from: https://www.argentina.gob.ar/coronavirus/vacuna/ preguntas-frecuentes-pediatricas [Last access: 2022-01-24].

12. Argentina. ANMAT. Disposición 450-2022; 2022. Available from: http://www.anmat.gov.ar/boletin_anmat/enero_2022/Dispo_450-22.pdf.

13. Walter EB, Talaat KR, Sabharwal C, et al. Evaluation of the BNT162b2 Covid-19 Vaccine in Children 5 to 11 Years of Age. N Engl J Med. 2022;386(1):35-46. Available from: 10.1056/NEJMoa2116298.

14. Argentina. Ministerio de Salud. Argentina firmó un nuevo acuerdo con Pfizer por 18,5 millones de vacunas para 2022 que incluye dosis pediátricas; 2022. Available from: https://www.argentina.gob.ar/noticias/argentina-firmo-un-nuevo-acuerdo-con-pfizer-por-185-millones-de-vacunas-para2022-que.

15. Frenck RW, Klein NP, Kitchin N, C4591001 Clinical Trial Group, et al. Safety, Immunogenicity, and Efficacy of the BNT162b2 Covid-19 Vaccine in Adolescents. N Engl J Med. 2021;385(3):239-250. Available from: 10.1056/NEJMoa2107456.

16. Ali K, Berman G, Zhou H, et al. Evaluation of mRNA-1273 SARS-CoV-2 Vaccine in Adolescents. N Engl J Med. 2021;385(24):2241-2251. Available from: 10.1056/NEJMoa2109522.

17. Xia S, Zhang Y, Wang Y, et al. Safety and immunogenicity of an inactivated COVID-19 vaccine, BBIBP-CorV, in people younger than 18 years: a randomised, double-blind, controlled, phase 1/2 trial. Lancet Infect Dis. 2021;(21):462-462. Available from: 10.1016/S1473-3099(21)00462-X.Epub.

18. Argentina. Ministerio de Salud de la Nación. CoNaSeVa. Campaña Nacional de Vacunación contra la COVID-19. Informe especial de vigilancia de seguridad en vacunas en niños, niñas y adolescentes. Ministerio de Salud de la Nación; 2021. Available from: https://bancos.salud.gob.ar/sites/ default/files/2021-11/Informe_especial_de_vigilancia_de_seguridad_en_vacunas_en_ninios_ninas_adolescentes.pdf.

19. Gargano JW, Wallace M, Hadler SC, et al. Use of mRNA COVID-19 Vaccine After Reports of Myocarditis Among Vaccine Recipients: Update from the Advisory Committee on Immunization Practices - United States, June 2021. MMWR Morb Mortal Wkly Rep. 2021;70(27):977-982. Available from: $10.15585 / \mathrm{mmwr}$ mm7027e2.

20. Sociedad Argentina de Pediatría. Documento de posición sobre vacunas contra COVID-19 en pediatría; $2021 . \quad$ Available from: https://www.saludneuquen.gob.ar/wp-content/uploads/2021/12/Documento-de-posici\%C3\%B3n-sobre-vacunas-contra-COVID19-en-pediatr\% C3\%ADa-SAP-30-11-21.pdf. 